\title{
Sensitivity Pattern of Micro Organisms of Septicemia in Neonatal Intensive Care Unit of a Tertiary Hospital, Bangladesh
}

\author{
Chowdhury CB MD ${ }^{1 *}$, Barua $\mathrm{S}^{2}$, Ferdous $\mathrm{J}^{3}$ and Chowdhury $\mathrm{N}^{4}$ \\ ${ }^{1}$ Department of Neonatology, Chittagong Medical College, Bangladesh \\ ${ }^{2}$ Department of Gynae \& Obs, Chittagong Medical College, Bangladesh \\ ${ }^{3}$ Department of Pediatrics, Chittagong Medical College Hospital, Bangladesh \\ ${ }^{4}$ Medical Officer, Chittagong Medical College Hospital, Bangladesh \\ Submission: October 01, 2016; Published: December 01, 2016 \\ *Corresponding author: Chiranjib Barua Chowdhury, Department of Neonatology, Chittagong Medical College, Bangladesh, \\ Email: barua.chiranjib@yahoo.com
}

\begin{abstract}
Neonatal septicemia is a clinical syndrome of systemic illness accompanied by bacteremia occurring in the first 28 days of life. Microorganism causing neonatal septicemia varies from country to country and region to region and time to time. In many situations, conventional antibiotics are not sensitive to causative micro-organism. Our aim of the study is to identify causative organism and sensitivity pattern of micro-organism in Neonatal Intensive Care Unit, (NICU)

Methods \& Subject: Total 300 patients were enrolled in this study with clinically diagnosed septicemia with prior antibiotic treatment or not. This study was done in NICU of tertiary Hospital, Chittagong Medical College, Bangladesh during the period of January 2013 to July 2014. Blood culture was done in Bact/Alerd 3D system.

Result: Out of 300 cases, 204 cases were culture negative and 96 cases (32\%) were culture positive. Klebsiella is the commonest causative organism 52(54.17\%), followed by Pseudomonas 16(16.67\%), Acinetobacter 14(14.58\%), S. aureus 6(6.25\%), E.coli 6(6.25\%), E. coli with other Coliform 2(2.08\%). In this study, sensitivity of Klebsiella was mostly to Ciprofloxacin, Imipenem, Azithromycin, Co-trimoxazole, Piperaciline and Tazobactam. Sensitivity to Pseudomonas was mostly to Amikacin, Imipenem, Ciprofloxacin, Azithromycin and Cefoperazone with Sulbactum.. Sensitivity to Acinetobactor was mostly to Amikacin, Imipenem, Ciprofloxacin, Netilmicin. Sensitivity to S. aureus was mostly to Vancomycine, Amikacin, Imipenem, Meropenem.. Sensitivity of E.coli was mostly to Amikacin, Ciprofloxacin, Ceftazidin. Most of the culture positive organisms not sensitive to conventional Ampicilin, Gentamycin.

Conclusion: Microorganisms identified causing neonatal septicemia in our region are Klebsiella, Pseudomonas, Acinetobactor, E. coli and Enterobector. Antibiotic sensitive to identified microorganism were Amikacin, Ciprofloxacin, Levofloxacin, Imipenem, Meropenem, Vancomycin and Ceftazidim..Conventional Ampicillin sensitivity is not detected and Gentamicin sensitivity is also low.

Keywords: Zinc; Malnutrition; Renal failure; Bone
\end{abstract}

\section{Introduction}

Neonatal septicemia is a clinical syndrome of systemic illness accompanied by bacteremia occurring in the first 28 days of life [1]. Micro-organisms causing neonatal septicemia are a bit different from region to region and also country to country depending upon various factors especially socioeconomic conditions and personal hygiene with environment. It may occur through transplacental infection or an ascending infection from mother's genitourinary tract and from the infected caregiver or nosocomial infections. The incidence of neonatal sepsis is 5 to 8 per 1000 birth, the highest rates occur in LBW, perinatal asphyxia, maternal infection and babies with congenital abnormalities [2]. In global perspective microorganisms most commonly associated with neonatal sepsis include groupB Streptococci, coagulase-Negative Staphylococci, Klebsiella, and Pseudomonas, Enterobacter, Candida, Streptococcus, anaerobes and non typeable $\mathrm{H}$ influnzae. It is important to note that $20-30 \%$ of the survivors 
of neonatal sepsis may exhibit neurological sequelae [2]. Sepsis related mortality is however largely preventable with rational antimicrobial therapy and aggressive supportive care. In most developing countries, gram-negative bacteria remain the major source of infection and gram positive microorganisms have been implicated in developed countries, as the most common cause of neonatal sepsis.While culture positive neonatal sepsis in the USA is $0.98 \%$ but in very low birth weight infants under prolonged intensive care the culture proven sepsis may beashigh as $30 \%$ [3]. Neonatal sepsis is one of the commonest cause of perinatal mortality in the developing world. Mortality rate of early onset neonatal sepsis is 2 to $40 \%$ and late onset neonatal sepsis is 2 to $20 \%$.Definitive diagnosis of neonatal sepsis is based on positive blood or cerebrospinal fluid (CSF) cultures, both of which take at least 24 to 48 hours and are often falsely negative. Due to irrational use of antibiotic, sensitivity pattern of microbes are also changed. In many situations, conventional antibiotics are not sensitive to causative micro-organisms \& developed increased drug resistance making treatment extremely difficult. Thus, local epidemiology of neonatal sepsis should be constantly updated to detect changes in the pattern of infective pathogens and their susceptibility to various antibiotics. Objectives of the study were to identify the causative organisms causing neonatal septicemia and to detect culture-sensitivity of the microorganisms.

\section{Methodology}

This is a hospital based prospective case series study which was performed in Neonatal intensive care unit (NICU), Chittagong Medical College Hospital, Bangladesh. Duration of this study was January 2013 to July 2014.and sample size was300. Inclusion Criteria were Term and preterm babies irrespective of sex and weight, clinically diagnosed neonatal septicemia patient under antibiotic treatment and also without, prior antibiotic therapy included in this study. Exclusion Criteria were severe perinatal asphyxia, neonate with congenital anomalies, extremely low birth weight neonates and respiratory distress syndrome.

A baby with good primitive reflex, activity and breast fed in initial 48 hours of life, who then became sick were considered as neonatal sepsis. Characteristic findings of neonatal sepsis patients were respiratory distress in the form of tachypnoea and or severe chest in drawing, reluctant to feeding, abdominal distension, lethargy, hypothermia, Sclerema, Convulsion. On the day of admission, from every case $3 \mathrm{ml}$ of blood was collected from peripheral vein with asceptic condition and introduced into aerobic and anaerobic media. The blood culture specimens were sent and processed according lyin Bact/ Alert 3D system in Microbiology laboratory. Departmental ethical committee gave consent to carry on this study and consent also taken from parents of this study cases.

\section{Result}

All the cases were grouped into three : Normal birth weight babies (NBW) (wt $\geq 2500$ g) was $30 \%$, Low birth weight,
LBW(1500-2500g) was 48\% main bulk of patient in the study cases and very low birth weight babies, VLBW( $<1500-1000 \mathrm{~g})$ was $22 \%$ (Figure: 1 ). In NBW group $57.8 \%$ were male and $42.2 \%$ were female. In LBW group 53\% were male whereas $47 \%$ were female. In VLBW group 52.8\% were male with $47.2 \%$ were female. Male female distribution was statistically insignificant. In NBW group early onset sepsis (EONS) was $42.2 \%$ but in late onset sepsis (LONS) was $57.8 \%$. In LBW group EONS was $68.2 \%$ but late onset Sepsis $31.8 \%$.

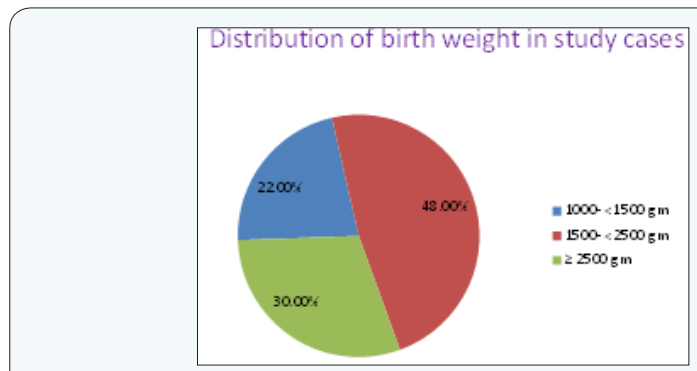

Figure: 1: Distribution of birth weight in study cases.

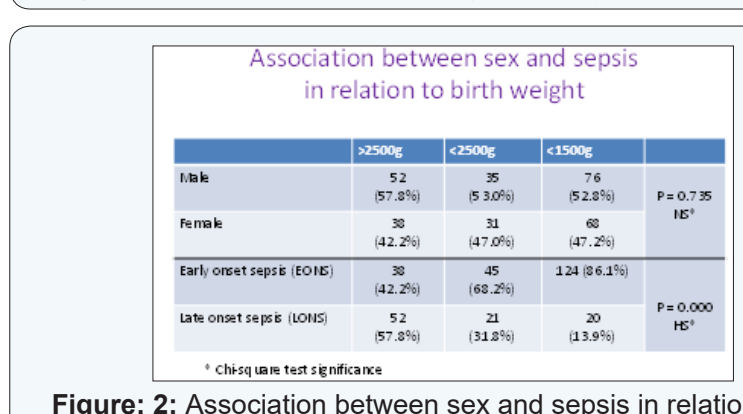

Figure: 2: Association between sex and sepsis in relation to birth weight.

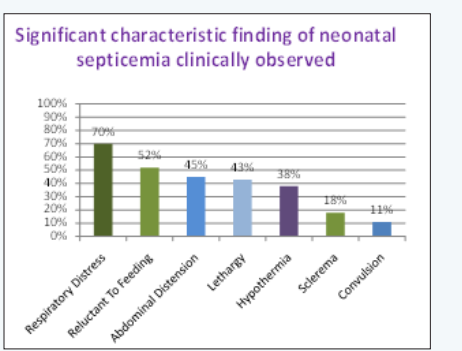

Figure: 3: Significant characteristic finding of neonatal septicemia clinically observed.

In VLBW group, EONS was $86.1 \%$ but in LONS was 13.9 (Figure 2). Characteristic clinical findings of neonatal sepsis patients were respiratory distress $70 \%$, reluctant to feeding (52\%), Abdominal distension (45\%),lethargy (43\%),hypothermia (38\%), Sclerema (18\%),Convulsion (11\% (Figure 3) Early onset sepsis was more common in study population which was statistically significant. Serial C-reactive protean is a highly sensitive marker for neonatal sepsis. In this study $78 \%$.of sepsis cases were positive for C-reactive protein but $100 \%$ of culture positive cases were positive for C-reactive protein. In this study, $32 \%$ cases were culture positive but $68 \%$ cases were culture negative which were clinically sepsis, out of 300 cases, culture positive only 96 (32\%), where Klebsiella (54.17\%), Pseudomonas Aeruginosa (16.67\%), Acinetobactor (14.58\%), staphylococcus 
aureus (6.25\%), Escherichia coli (6.25\%) with mixed infection Escherichia coli with coliform $(2.08 \%)$ (Figure 4). Culture sensitivity of Klebsiella to ciprofloxacin (78\%) Imipenem (65\%) Levofloxacin (50\%), Meropenem (45\%), Cefotaxime(40\%), Ceftazidim (38\%) (Figure 5). Sensitivity of pseudomonas to (69\%), Ceftazadim (50\%) and Ciprofloxacin (45\%) (Figure 6). It was also observed that sensitivity of Acinetobactor to Amikacin (81\%) Ciprofloxacin (65\%), Imipenem (58\%), Meropenem (55\%) and Netilmycin (60\%) (Figure 7) respectively. Culture sensitivity of staphylococcus aureus to Vancomycin (83\%), followed by Amikacin 60\%, Meropenem (60\%), Imipenem (55\%) and Gentamicin (48\%) (Figure 8). Culture sensitivity of E. coli to Amikacin (87\%), Ciprofloxacin (75\%) and Ceftazidim (55\%) (Figure 9).

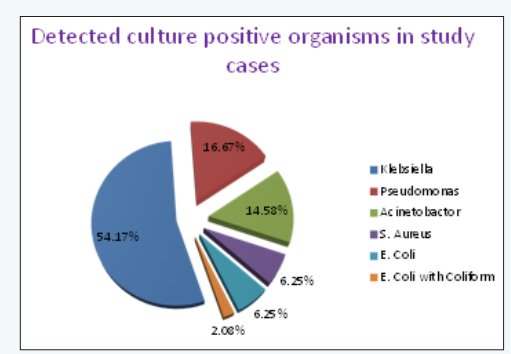

Figure: 4: Detected culture positive organisms in study cases.
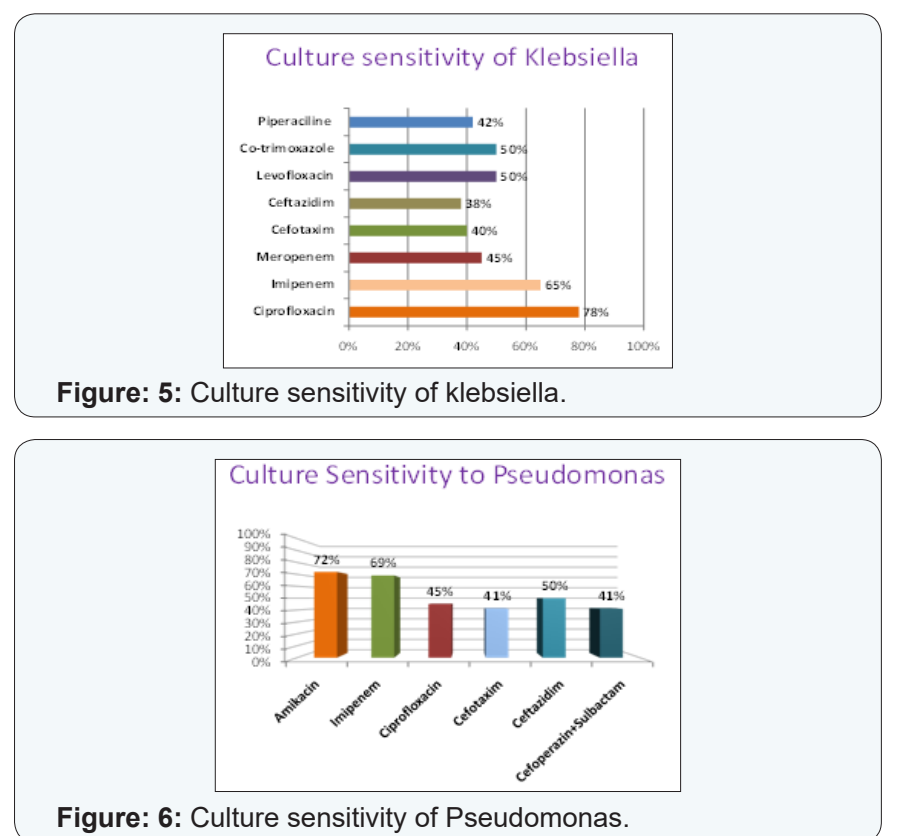

Figure: 6: Culture sensitivity of Pseudomonas.

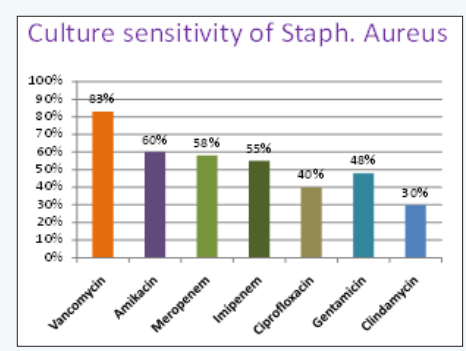

Figure: 7: Culture sensitivity of Staph. Aureus.

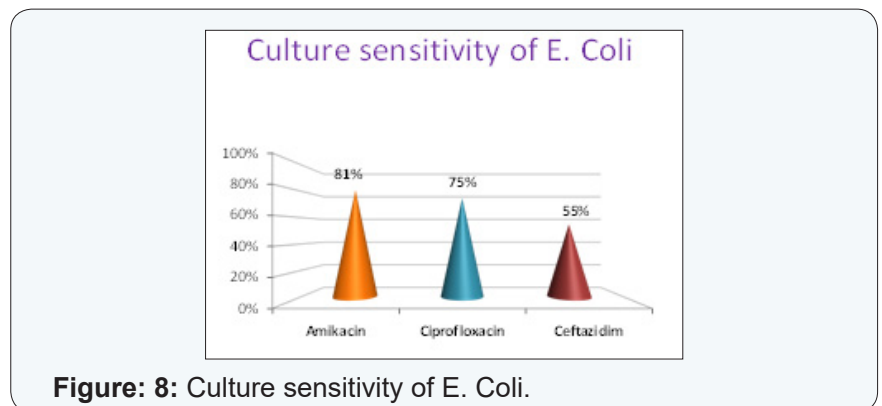

Figure: 8: Culture sensitivity of E. Coli.

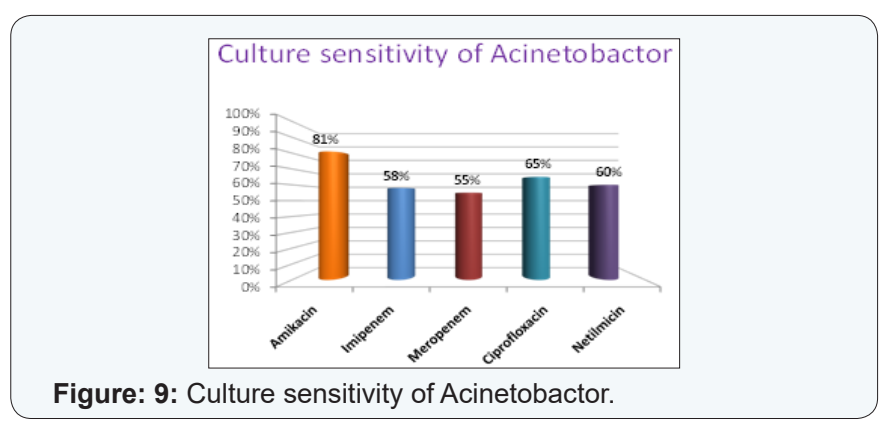

\section{Discussion}

The cases were grouped into three:- Normal birth weight (NBW) were 30\%, 48\% were low birth weight (LBW) and 22\% were very low birth weight (VLBW) babies. LBW (70\%) babies were vulnerable to neonatal sepsis which is similar to other studies. Early onset neonatal sepsis (EONS) was more common than late onset which is consistent with other studies. In this study $78 \%$ cases were positive for C-reactive protein but $100 \%$ were positive in culture positive cases. Study of west B. A [4] in port Hurcourt Nigeria observed that Klebsiella pneumonia (50\%), staphylococcus aureus (21\%) were predominant microbes and sensitive to Ciprofloxacin $88.8 \%$, 99.8\% respectively but in our study Klebsiella pneumonia (54.17\%), pseudomonas aeruginosa $(16.67 \%)$ were predominant microbes and staphylococcus aureus (6.25\%) but Klebsiella was mostly sensitive to ciprofloxacin (78\%). In the study of Mehroety et al. [5] in Turki found that staphylococcus aureus (44\%), Klebsiella (10.5\%) were predominant microbes and sensitive to vancomycine and carbapenem, ciprofloxacin respectively. They noticed fungal infection was $19.2 \%$ in their study. Sensitivity pattern is also similar to our study but pattern of organisms varies. Shah and Desai [6] observed in their study E. coli with Klebsiella pneumonia were predominant organisms caused neonatal sepsis but in our study Klebsiella with pseudomonas were the predominant organisms and most of the gram negative organisms were sensitive to Amikacin, Imipenem and gram Combined drugs piperacillin and tazobactum, which were consistent to our study but we observed along with above drugs ciprofloxacin and Levofloxacin were also sensitive. Ramesh \& Lincy [7] found in their study $28.8 \%$ cases were blood culture positive and common isolated organisms were Klebsiella followed by pseudomonas and higher resistance to the commonly used 1st line antibiotics Ampicillin and gentamicin. 
Our study almost consistent to this study. Samia, et al. [8] where identified negative organism were the most common pathogens, $80 \%$, were Klebsiella pseudomonas (35\%) Enterobator (2\%) and gram positive organisms were staphylococcus $10 \%$ and GBS $10 \%$. But in our study, GBS not detected in any blood culture growth. In the study of Viwanathan, et al. [9] found in their study gramnegative organisms were the main causes of neonatal sepsis and those organisms were almost $100 \%$ resistent to conventional Ampicillin and Gentamicin. Their study findings were similar to our study. In the study of Sharifun \& Afroza, et al. [10] at Dhaka, Bangladesh observe Pseudomonas, E-coli and coagulase negative staph common pathogens of neonatal sepsis but in our study pseudomonas, Klebsiella, AcinetobactorE-coli were common pathogens but Anti biotic sensitivity almost similar. In the study of Sharma \& Agarwal [11] isolated organisms were staphauras, Klesiella, E-Coli and their study also mimic to this study. Staphylococcus auras Agarwel \& Bhat [12]. Observed that $32.72 \%$ were blood culture positive and common bacteria isolated were Klebsiella, E-coli and Staphylococcus aureus. No single group B-streptococcus. (GBS) growth found in blood culture.Limitation of this study was small size sample from one NICU \& Blood culture done from a number of cases after antibiotic therapy. There was no conflict of interest. We acknowledge Principal, Director of this institute and neonates who gave opportunity to carry out this study.

\section{Conclusion}

Microorganisms identified causing neonatal septicemia in our region are Klebsiella, Acinetobactor, E. coli and Enterobector. Antibiotics sensitive to identified micro organism were Amikacin, Ciprofloxacin, Levofloxacin, Imipenem, Meropenem, Vancomycin, Ceftazidim. Conventional Ampicillin sensitivity is not detected and Gentamicin sensitivity is also low. Multi centre broad based study for blood culture sensitivity is essential to guide antibiotic regime for neonatal septicemia in developing and developed countries.

\section{References}

1. Gotoff SP (2016) Infection of the neonatal infant. In: Behrman RE Kliegman RM, Jenson HB. Nelson textbook of Pediatrics. $16^{\text {th }}$ (edn) Philadelphia: WB Saunders Company eds pp. 909-925.

2. Mery TC (2009) Neonatal sepsis. National Institute of Health, Online Medical Library.

3. Stoll BJ, Hansen NI, SanchjebPJ (2011) Early onset neonatal Sepsis. Journal of Pediatrics.

4. West BA, Peterside $O$ (2012) Sensitivity Pattern among bacteria isolets in Neonatal Septicemia in Port Hurcout. Ann Clin Microbiol Antimicrob 11: 17.

5. Yalaz M, Cetin H, Akisu M, Aydemir S, Tunger A, et al. (2006) Neonatal nosocomial sepsis in level 111 NICU. Evaluation of the causative agents and antimicrobial susceptibilities. Turk J Pediatr 48(1): 13-18.

6. Shah M, Desai P (2015) Current Scenario of antimicrobial resistance in NICUS. An emerging therapeutic confront. International Journal of Analytical, Pharmacological and Biomedical Sciences J. International medicine and Dentistry 2(1): 22-29.

7. Ramesh BY, Lincy PB (2011) Early Onset of Neonatal Sepsis: Analysis of the Risk Factors and the Bacterial Isolates by Using the BacT Alert System. Journal of clinical and Diagnostic Research 5(7): 1385-1388.

8. Samia B, Iman S, Gams LS (2011) Journal of American Scienc 7(7).

9. Viswanathan R, Singh AK, Ghosh C, Dasgupta S, Mukherjee S, et al (2012) profile of neonatal septicemia at district level sick newborn care unit. J Health Popul Nutr 30(1): 41-48.

10. Naher BS, Afroza S, Roy S, Nahar N, Kunda TN (2013) Neonatal Sepsis in a tertiary care hospital: Evaluation of causative agents and antimicrobial susceptibilities. Bangladesh Journal of Child Health $37(1)$.

11. Sharma C M, Agrawal RP, Sharma H, Kumar B, Sharma D, et al. (2013) "Neonatal Sepsis": Bacteria and their Susceptibility pattern towards Antibiotics in neonatal intensive care unit. J Clin Diagn Res 2013 7(11): $2511-2513$.

12. Agarwal A, Bhat S (2015) Clinicobiological study of neonatal sepsis. Journal of international Medicine and Dentistry 2(1): 20-29.

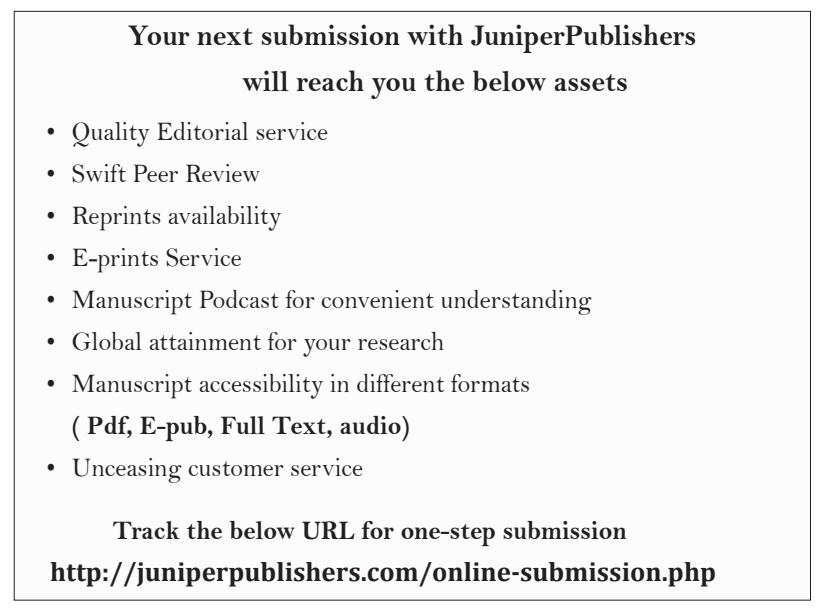

